\title{
The two faces of static correlation
}

\author{
Joshua W. Hollett ${ }^{\text {a) }}$ and Peter M. W. Gill ${ }^{\text {b) }}$ \\ Research School of Chemistry, Australian National University, Canberra ACT 0200, Australia
}

(Received 18 January 2011; accepted 4 March 2011; published online 21 March 2011)

\begin{abstract}
Restricted Hartree-Fock (RHF) and UHF wavefunctions for beryllium-like ions with nuclear charge $3 \leq Z \leq 5$ are found using a near-complete Slater basis set. The triplet (RHF $\rightarrow$ UHF) instability and correlation energy are investigated as a function of $Z$ and we find that the instability vanishes for $Z>4.5$. We reproduce this surprising behavior using a minimal-basis model and, by comparing with the stretched $\mathrm{H}_{2}$ molecule, conclude that "static" (also known as nondynamical, near-degeneracy, first-order, or strong) correlation comes in two flavors: one that can be captured by UHF and another that cannot. In the former (Type A), there is an "absolute near-degeneracy"; in the latter (Type B), there is a "relative near-degeneracy." This dichotomy clarifies discussions of static correlation effects. (C) 2011 American Institute of Physics. [doi:10.1063/1.3570574]
\end{abstract}

\section{INTRODUCTION}

Quantum chemical calculations have become routine for a wide variety of chemical species but, because of the complexity of electron correlation [i.e., phenomena lying beyond the Hartree-Fock (HF) approximation], such calculations inevitably strike a compromise between accuracy and efficiency. The correlation problem is usually treated using either post-HF approaches ${ }^{1}$ (e.g., configuration interaction or perturbation theory) or density functional methods ${ }^{2}$ and, although new approaches (such as density matrix functional theory, ${ }^{3-5}$ intracule functional theory, ${ }^{6-10}$ the density matrix renormalization group, ${ }^{11-14}$ and quantum Monte Carlo ${ }^{15,16}$ ) are developing, there is still no universally accurate and efficient approach. In order to progress toward that goal, it is essential that our understanding of the nature of electron correlation become more precise and sophisticated.

Löwdin defined ${ }^{17}$ the correlation energy,

$$
E_{\text {corr }}=E_{\text {exact }}-E_{\mathrm{RHF}},
$$

as the difference between the exact nonrelativistic energy and the restricted Hartree-Fock (RHF) energy in a complete basis set. When studying correlation effects and developing methods to treat them, many workers have found it useful to subdivide further and write ${ }^{18}$

$$
E_{\text {corr }}=E_{\text {dyn }}+E_{\text {stat }}
$$

where the dynamic correlation energy $E_{\text {dyn }}$ arises from the inability of HF theory to model interelectronic cusps ${ }^{19}$ and dispersion interactions, ${ }^{20,21}$ and the static (also called nondynamical, near-degeneracy, left-right, or first-order) correlation energy $E_{\text {stat }}$ arises from near-degeneracies of the HartreeFock occupied and virtual orbitals. Systems with significant $E_{\text {stat }}$ are poorly described by a Slater determinant and are said to have multireference character. Typically, they have stretched or multiple bonds, partially occupied degenerate orbitals, or are electronically excited. ${ }^{22}$

\footnotetext{
a)Electronic mail: jhollett@rsc.anu.edu.au.

b)Electronic mail: peter.gill@anu.edu.au.
}

The simplest system with significant $E_{\text {stat }}$ is the stretched $\mathrm{H}_{2}$ molecule. As the bond lengthens, the $\operatorname{HOMO}\left(\sigma_{g}\right)$ and LUMO $\left(\sigma_{u}\right)$ become degenerate, one electron settles around each nucleus, and $E_{\text {corr }}$ approaches a limiting value that reflects the inability of the RHF model to describe this localization. Analogous behavior is observed for the homolytic dissociation of any bond. Static correlation is found in some systems even at their equilibrium geometries. In $\mathrm{O}_{3}$, for example, the HOMO-LUMO gap is small, the molecule is significantly biradicaloid, ${ }^{23,24}$ and $E_{\text {stat }}$ is a large fraction of $E_{\text {corr }}{ }^{25}$

Static correlation is also found in small-gap atomic systems. The simplest example is the ground-state of the Be atom where, because the $2 s$ and $2 p$ orbitals are nearly degenerate, one must include both the $1 s^{2} 2 s^{2}$ and $1 s^{2} 2 p^{2}$ configurations to describe the state accurately. ${ }^{26-30}$ If the nuclear charge $Z$ is varied in nonrelativistic, fixed-nucleus calculations, one finds ${ }^{31-33}$ that (in atomic units)

$$
\begin{aligned}
& E_{\text {exact }}^{\mathrm{Be}}=-\frac{5}{4} Z^{2}+1.55927 Z-0.8775+O\left(Z^{-1}\right), \\
& E_{\mathrm{RHF}}^{\mathrm{Be}}=-\frac{5}{4} Z^{2}+1.57100 Z-0.8055+O\left(Z^{-1}\right),
\end{aligned}
$$

and therefore the correlation energy,

$$
E_{\text {corr }}^{\mathrm{Be}}=-0.01173 Z-0.0720+O\left(Z^{-1}\right)
$$

grows linearly with $Z$. Such linear growth is a consequence of the near-degeneracy ${ }^{31}$ and may be contrasted, for example, with the qualitatively different behavior,

$$
\begin{aligned}
& E_{\mathrm{corr}}^{\mathrm{He}}=-0.0467+O\left(Z^{-1}\right), \\
& E_{\mathrm{corr}}^{\mathrm{Sp}}=-0.0476+O\left(Z^{-1}\right), \\
& E_{\mathrm{corr}}^{\mathrm{Ho}}=-0.0497+O\left(Z^{-1}\right), \\
& E_{\mathrm{corr}}^{\mathrm{Ba}}=-0.0552+O\left(Z^{-1}\right),
\end{aligned}
$$

of various two-electron systems (He-like ions, spherium, hookium, and ballium ${ }^{34-37}$ in which the HOMO and LUMO remain well-separated as $Z$ grows. 
Although static correlation arises from multireference character, it can be partially modeled by relaxing the constraint that the $\alpha$ and $\beta$ spin orbitals be equivalent. The resulting unrestricted Hartree-Fock (UHF) wavefunction, which is still a single determinant but no longer an eigenfunction of the spin-squared operator $\boldsymbol{S}^{\mathbf{2}}$, is able to capture a fraction of $E_{\text {stat }}$ and thus yield a lower total energy. ${ }^{38,39}$ The existence of such a lower-energy UHF wavefunction, i.e.,

$$
\Delta E_{\mathrm{UHF}}=E_{\mathrm{RHF}}-E_{\mathrm{UHF}}>0,
$$

in a singlet system is known as a triplet instability ${ }^{40}$ and, in the case of $\mathrm{H}_{2}$, it is present whenever the bond length exceeds 2.296 bohr. $^{41}$ As the bond stretches beyond this, the $\mathrm{UHF}$ wavefunction captures progressively more of $E_{\text {stat }}$ until, at the dissociation limit, it yields the exact energy. ${ }^{1}$ A less dramatic example is found in $\mathrm{O}_{3}$ at its equilibrium geometry, where UHF recovers $10 \%$ of the correlation energy. ${ }^{42}$

The ability of UHF to capture part of the static correlation led Pople to propose the alternative definition ${ }^{43}$

$$
E_{\text {corr }}=E_{\text {exact }}-E_{\mathrm{HF}},
$$

where $E_{\mathrm{HF}}$ is the lowest energy of any single-determinant wavefunction. To the extent that they avoid anomalous behavior in multireference systems, Pople correlation energies may be more robust than Löwdin ones. ${ }^{44}$

Given the multireference character of the Be-like ions, it is not surprising to find that Be itself possesses a triplet instability. ${ }^{45}$ Moreover, in the higher Be-like ions where the correlation energy (5) increases rapidly with $Z$, we would expect $\Delta E_{\mathrm{UHF}}$ also to increase.

In this paper, we critically examine this expectation, computing small- and large-basis RHF and UHF wavefunctions for the Be-like ions. In Sec. II, we study the triplet instability in a near-complete basis and, in Sec. III, we show that the key features are preserved in a minimal basis. In Sec. IV we discuss the ability of UHF to capture $E_{\text {stat }}$ and we propose a new categorization of static correlation energies. The presence of different forms of static correlation is then analyzed in both the Be-like ions and $\mathrm{H}_{2}$. Atomic units are used throughout.

\section{LARGE-BASIS CALCULATIONS}

RHF and UHF wavefunctions of $\mathrm{Li}^{-}, \mathrm{Be}$, and $\mathrm{B}^{+}$, as well as the hypothetical Be-like ions $\mathrm{A}^{(Z-4)}$, where $Z=3.25$, $3.5,3.75,4.25,4.5$, and 4.75 , were found by solving the Roothaan-Hall $^{46,47}$ and Pople-Nesbet ${ }^{38}$ equations by iterative diagonalization in MATHEMATICA, ${ }^{48}$ terminating when the energy changed by less than $10^{-10}$. We employed a scaled universal even-tempered Slater-type $s p$ basis set, ${ }^{49-51}$ adding diffuse functions via the Cooper-Wilson protocol ${ }^{52}$ for the negative ions. Only $s$ functions are needed to describe the RHF orbitals, but $p$ functions are required to give the lower-symmetry UHF orbitals, when they exist. UHF orbitals were found by mixing the RHF HOMO $(2 s)$ and LUMO $\left(2 p_{z}\right)$ and then performing a UHF calculation within $C_{\infty v}$ symmetry.

For each $Z$, the basis was systematically extended until the total energy converged to six decimal places, thus obtaining the $s p$-limit HF energies to within 1 microhartree.
TABLE I. $E_{\mathrm{RHF}}\left(E_{\mathrm{h}}\right), \Delta E_{\mathrm{UHF}}\left(\mathrm{m} E_{\mathrm{h}}\right)$, and $E_{\text {corr }}\left(\mathrm{m} E_{\mathrm{h}}\right)$ of Be-like ions with $3 \leq Z \leq 5$.

\begin{tabular}{lrcr}
\hline \hline$Z$ & $-E_{\mathrm{RHF}}$ & $\Delta E_{\mathrm{UHF}}$ & $-E_{\text {corr }}{ }^{\mathrm{a}}$ \\
\hline 3.00 & 7.428232 & 2.767 & 72.541 \\
3.25 & 8.976184 & 2.013 & \\
3.50 & 10.683680 & 1.268 & \\
3.75 & 12.549514 & 0.688 & \\
4.00 & 14.573023 & 0.294 & 94.333 \\
4.25 & 16.753807 & 0.072 & \\
4.50 & 19.091604 & 0.000 & \\
4.75 & 21.586235 & 0 & 111.308 \\
5.00 & 24.237575 & 0 & \\
\hline \hline
\end{tabular}

${ }^{a}$ Reference 29

Table I lists our RHF energies and UHF stabilizations $\Delta E_{\mathrm{UHF}}$, and a few $E_{\mathrm{corr}}$ values from Komasa et al. ${ }^{29}$ Our RHF energies agree perfectly with those of Koga et al. ${ }^{53}$ for $\mathrm{Li}^{-}$, $\mathrm{Be}$, and $\mathrm{B}^{+}$, confirming the quality of our basis.

Like Ivanov, ${ }^{45,54}$ we find that the Be atom itself has a triplet instability. However, we find that $\Delta E_{\mathrm{UHF}}$ decreases from $2.767 \mathrm{~m} E_{\mathrm{h}}$ at $Z=3$ to zero at $Z=4.5$. As a consequence, none of the real four-electron cations ( i.e., $\mathrm{B}^{+}$, $\mathrm{C}^{2+}, \ldots$ ) has a triplet instability. Contrary to our expectations, the growth in $E_{\text {corr }}$ with $Z$ is not accompanied by an increase in $\Delta E_{\mathrm{UHF}}$. Indeed, UHF is unable to capture any correlation energy at all for $Z>4.5$.

\section{MINIMAL-BASIS CALCULATIONS}

Can the instability behavior in the Be-like ions be understood without resorting to near-complete basis sets? To answer this, we repeated our study using the minimal orthonormal Slater RHF orbitals,

$$
\begin{gathered}
\psi_{1}(\boldsymbol{r})=\sqrt{a^{3} / \pi} \exp (-a r) \\
\psi_{2}(\boldsymbol{r})=\sqrt{\frac{b^{5}(a+b)^{2}}{3 \pi\left(a^{2}-a b+b^{2}\right)}\left[\frac{3}{a+b}-r\right] \exp (-b r),} \\
\psi_{3}(\boldsymbol{r})=\sqrt{b^{5} / \pi} z \exp (-b r), \\
\psi_{4}(\boldsymbol{r})=\sqrt{b^{5} / \pi} y \exp (-b r) \\
\psi_{5}(\boldsymbol{r})=\sqrt{b^{5} / \pi} x \exp (-b r) .
\end{gathered}
$$

Using the usual one- and two-electron integrals,

$$
\begin{gathered}
h_{a a}=\int \psi_{a}(\boldsymbol{r})\left[-\frac{\nabla^{2}}{2}-\frac{Z}{r}\right] \psi_{a}(\boldsymbol{r}) d \boldsymbol{r}, \\
J_{a b}=\iint \psi_{a}\left(\boldsymbol{r}_{1}\right) \psi_{a}\left(\boldsymbol{r}_{1}\right) r_{12}^{-1} \psi_{b}\left(\boldsymbol{r}_{2}\right) \psi_{b}\left(\boldsymbol{r}_{2}\right) d \boldsymbol{r}_{1} d \boldsymbol{r}_{2}, \\
K_{a b}=\iint \psi_{a}\left(\boldsymbol{r}_{1}\right) \psi_{b}\left(\boldsymbol{r}_{1}\right) r_{12}^{-1} \psi_{a}\left(\boldsymbol{r}_{2}\right) \psi_{b}\left(\boldsymbol{r}_{2}\right) d \boldsymbol{r}_{1} d \boldsymbol{r}_{2},
\end{gathered}
$$


one can write the RHF energy as

$$
E_{\mathrm{RHF}}=2 h_{11}+2 h_{22}+J_{11}+J_{22}+4 J_{12}-2 K_{12} .
$$

To study the correlation energy and its capture by UHF, we now adopt the frozen-core approximation.

The full CI treatment uses a parameter $\omega$ to write

$$
\Psi_{\mathrm{CI}}=\Psi_{\mathrm{RHF}} \cos \omega-3^{-1 / 2}\left(\Psi_{22}^{33}+\Psi_{22}^{44}+\Psi_{22}^{55}\right) \sin \omega,
$$

mixing the RHF determinant with doubly substituted determinants wherein the $2 s$ pair is promoted into the $2 p_{x}, 2 p_{y}$, or $2 p_{z}$ orbital. Diagonalizing the resulting CI matrix yields the full CI energy,

$$
\begin{gathered}
E_{\mathrm{CI}}=E_{\mathrm{RHF}}-p \sin \omega \cos \omega+q \sin ^{2} \omega, \\
p=2 \sqrt{3} K_{23}, \\
q=2 h_{33}-2 h_{22}-4 J_{12}+4 J_{13}-J_{22}+J_{33} \\
+2 K_{12}-2 K_{13}+2 K_{34},
\end{gathered}
$$

where the parameter satisfies,

$$
\tan 2 \omega=\frac{p}{q}
$$

The UHF treatment uses a parameter $\theta$ and a perturbing orbital $\psi_{\mathrm{U}}$ to form the broken-symmetry orbitals,

$$
\begin{aligned}
& \psi_{1}^{\alpha}=\psi_{1}, \quad \psi_{2}^{\alpha}=\psi_{2} \cos \theta+\psi_{\mathrm{U}} \sin \theta, \\
& \psi_{1}^{\beta}=\psi_{1}, \quad \psi_{2}^{\beta}=\psi_{2} \cos \theta-\psi_{\mathrm{U}} \sin \theta,
\end{aligned}
$$

and these yield the UHF energy,

$$
\begin{aligned}
E_{\mathrm{UHF}}= & E_{\mathrm{RHF}}-2 P \sin ^{2} \theta+Q \sin ^{4} \theta \\
P= & h_{22}-h_{\mathrm{UU}}+2 J_{12}-2 J_{1 \mathrm{U}}+J_{22}-J_{2 \mathrm{U}} \\
& -K_{12}+K_{1 \mathrm{U}}+2 K_{2 \mathrm{U}} \\
& \\
Q= & J_{22}-2 J_{2 \mathrm{U}}+J_{\mathrm{UU}}+4 K_{2 \mathrm{U}}>0
\end{aligned}
$$

where the parameter satisfies

$$
\sin ^{2} \theta=\frac{P}{Q},
$$

where $P / Q$ is a dimensionless quantity. Thus, a triplet instability exists if and only if, $P>0$.

For simplicity, we initially chose the perturbing orbital,

$$
\psi_{\mathrm{U}}=\psi_{3},
$$

and then, for each $Z$ value, we determined the Slater exponents in Eq. (9) that minimize the RHF energy (13). Table II shows the resulting exponents, RHF, UHF lowering, and correlation energies. Figure 1 shows the decay of $\Delta E_{\mathrm{UHF}}$ with $Z$, for both basis sets. Although the $E_{\text {corr }}$ values in Table II are only half as large as those in Table I, they still grow linearly with $Z$. Moreover, as in the large basis, we see again that $\Delta E_{\mathrm{UHF}}$ decreases rapidly as $Z$ grows and, by solving the critical equation $P=0$, we find that the triplet instability disappears for $Z>4.138$. Although this critical value is slightly
TABLE II. Slater exponents, $E_{\mathrm{RHF}}\left(E_{\mathrm{h}}\right), \Delta E_{\mathrm{UHF}}\left(\mathrm{m} E_{\mathrm{h}}\right)$, and $E_{\text {corr }}\left(\mathrm{m} E_{\mathrm{h}}\right)$ of Be-like ions with $3 \leq Z \leq 5$.

\begin{tabular}{lccccc}
\hline \hline$Z$ & $a$ & $b$ & $-E_{\mathrm{RHF}}$ & $\Delta E_{\mathrm{UHF}}$ & $-E_{\text {corr }}$ \\
\hline 3.00 & 2.696 & 0.545 & 7.394756 & 3.642 & 27.712 \\
3.25 & 2.950 & 0.710 & 8.939821 & 2.010 & 32.320 \\
3.50 & 3.203 & 0.866 & 10.644745 & 0.932 & 36.340 \\
3.75 & 3.456 & 1.015 & 12.508374 & 0.310 & 40.030 \\
4.00 & 3.708 & 1.160 & 14.530009 & 0.035 & 43.514 \\
4.25 & 3.959 & 1.301 & 16.709193 & 0 & 46.865 \\
4.50 & 4.211 & 1.439 & 19.045616 & 0 & 50.122 \\
4.75 & 4.462 & 1.575 & 21.539059 & 0 & 53.314 \\
5.00 & 4.713 & 1.710 & 24.189363 & 0 & 56.458 \\
\hline \hline
\end{tabular}

smaller than it was in the large basis, it reveals the same unexpected feature: as $E_{\text {stat }}$ grows, UHF recovers less of it.

Alternatively, and more generally, we can assume that $Z$ is large and that the orbitals take the form,

$$
\begin{gathered}
\psi_{1}=\phi_{100}, \\
\psi_{2}=\phi_{200}, \\
\psi_{\mathrm{U}}=\sum_{n l m}^{\infty} c_{n l m} \phi_{n l m},
\end{gathered}
$$

where the $\phi_{n l m}$ are the standard hydrogenic orbitals ${ }^{55}$ for a nuclear charge $Z$ and, because $\psi_{\mathrm{U}}$ must be orthogonal to $\phi_{100}$ and $\phi_{200}$, the sum in Eq. (25c) does not include these. There are then two cases to consider. If $c_{n l m}=0$ for $n \geq 3$, it can be shown that

$$
P=-\frac{10013}{419904} Z
$$

Otherwise, the Schwarz inequality ${ }^{56} K_{a b} \leq J_{a b}$ yields

$$
\begin{aligned}
P & <h_{22}-h_{\mathrm{UU}}+2 J_{12}-J_{1 \mathrm{U}}+J_{22}+J_{2 \mathrm{U}}-K_{12} \\
& =-\left(\frac{1}{8}-\sum_{n l m}^{\infty} \frac{c_{n l m}^{2}}{2 n^{2}}\right) Z^{2}+O(Z) .
\end{aligned}
$$

In either case, it is clear that $P<0$ and therefore there is no triplet instability.

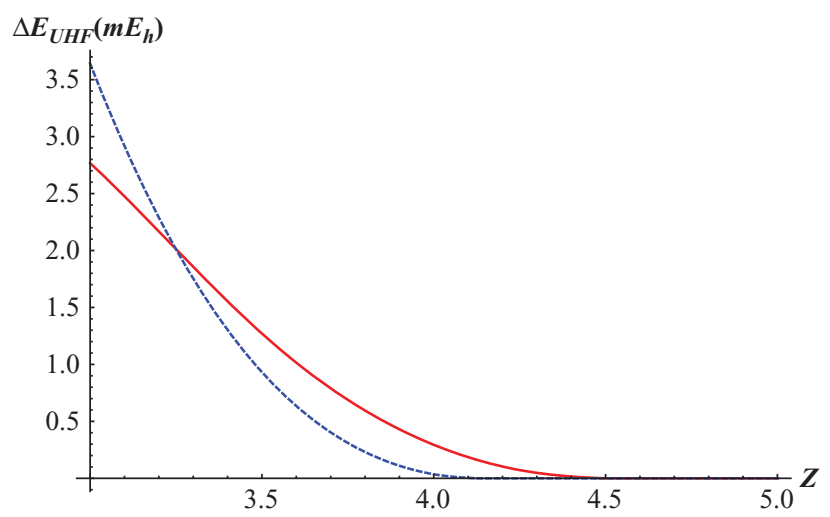

FIG. 1. $\Delta E_{\mathrm{UHF}}$ for the Be-like ions with $3 \leq Z \leq 5$ using the large (solid) and minimal (dashed) basis sets. 
TABLE III. HOMO and LUMO energies $\left(E_{\mathrm{h}}\right)$, in $\mathrm{H}_{2}$ molecule (bond length $=R$ ) and Be-like ions (nuclear charge $=Z$ ).

\begin{tabular}{|c|c|c|c|c|c|c|c|}
\hline \multicolumn{4}{|c|}{ Stretched $\mathrm{H}_{2}{ }^{\mathrm{a}}$} & \multicolumn{4}{|c|}{ Be-like ions } \\
\hline$R$ & $\epsilon_{\text {HOMO }}$ & $\epsilon_{\text {LUMO }}$ & $\Delta \epsilon$ & $Z$ & $\epsilon_{\mathrm{HOMO}}$ & $\epsilon_{\text {LUMO }}$ & $\Delta \epsilon$ \\
\hline 2 & -0.514 & +0.088 & 0.602 & 6 & -1.694 & -0.865 & 0.829 \\
\hline 4 & -0.380 & -0.058 & 0.322 & 8 & -4.091 & -2.816 & 1.275 \\
\hline 6 & -0.325 & -0.132 & 0.193 & 10 & -7.491 & -5.777 & 1.713 \\
\hline 8 & -0.297 & -0.164 & 0.134 & 12 & -11.892 & -9.743 & 2.149 \\
\hline 10 & -0.281 & -0.178 & 0.102 & 14 & -17.294 & -14.712 & 2.582 \\
\hline
\end{tabular}

${ }^{\mathrm{a}} \mathrm{RHF} / \mathrm{cc}-\mathrm{pV} 5 \mathrm{Z}$

\section{DISCUSSION}

Although $E_{\text {stat }}$ is large in the large- $Z$ Be-like ions, we have found that UHF theory is unable to capture any of it. This result is in stark contrast to highly stretched $\mathrm{H}_{2}$, where UHF recovers nearly all of $E_{\text {stat }}$. We conclude that there are two types of static correlation: Type A which can be captured by UHF and Type B which cannot.

We argue that the type of static correlation in a system depends on whether it has orbitals with an absolute neardegeneracy or a relative near-degeneracy. In other words, is the gap small in absolute terms or is it small only in comparison to the orbital energies?

In highly stretched $\mathrm{H}_{2}$, the HOMO-LUMO gap $\Delta \epsilon$ approaches zero as $R \rightarrow \infty$ (Table III). This absolute neardegeneracy produces Type A static correlation, which can be captured by UHF. In the Be-like ions, however, the gap actually widens as $Z$ increases (Table III) and the absence of an absolute near-degeneracy eventually prevents UHF from capturing any static correlation. However, there is still a relative near-degeneracy, because the orbital energies themselves grow quadratically (Table III) and this produces Type B static correlation which grows linearly with $Z$.

Figure 2 shows a decomposition of $E_{\text {corr }}$ in the Be-like ions as a function of $Z$. In region $\mathrm{I}$, where $Z<4.138$, there is Type D (dynamic), Type A, and Type B correlation but, as Table II shows, the Type A correlation is a small fraction (at most $13 \%$ ) of the total. In region II, the Type A correlation vanishes completely and UHF can no longer capture any of the correlation energy. Moreover, as $Z$ increases, Type B cor-

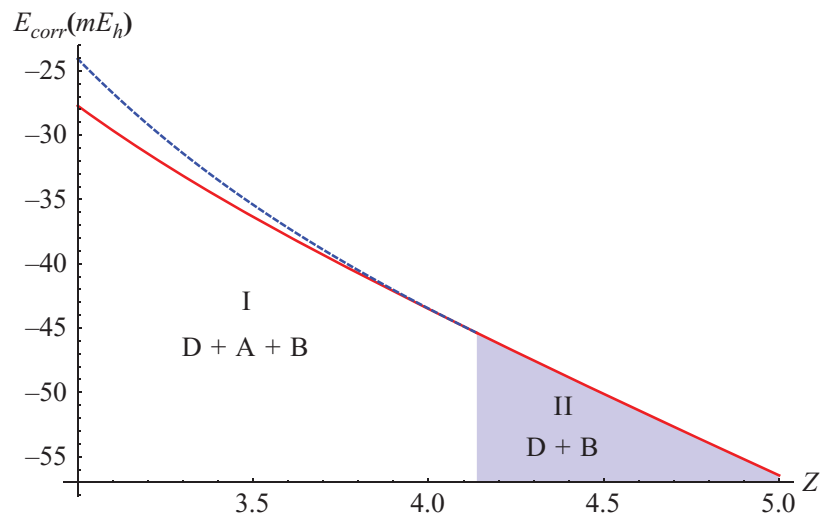

FIG. 2. Löwdin (solid) and Pople (dashed) correlation energies of Be-like ions using the minimal Slater basis set (9) showing regions where one finds Type A, B, and D correlation.

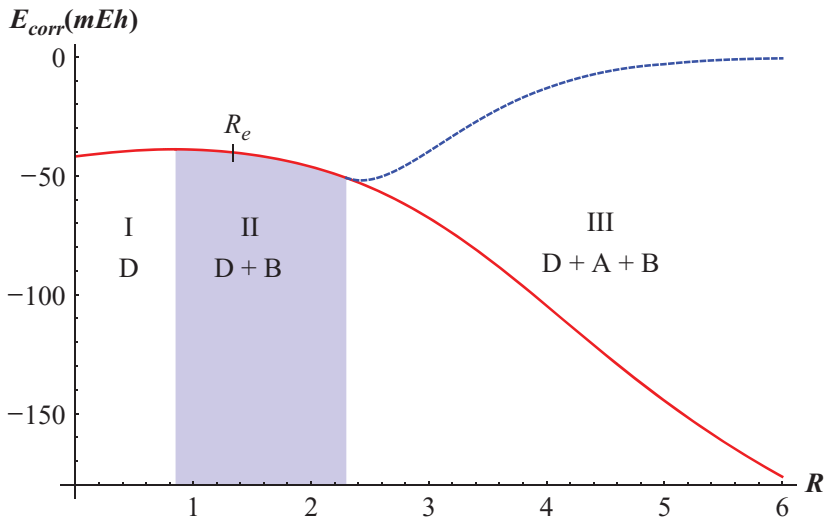

FIG. 3. Löwdin (solid) and Pople (dashed) correlation energies of $\mathrm{H}_{2}$ using the cc-pV5Z basis set, showing regions where one finds Type A, B, and D correlation.

relation becomes dominant because, as in the He-like ions, ${ }^{34}$ the Type D correlation approaches a constant.

Figure 3 shows an analogous decomposition of $E_{\text {corr }}$ in $\mathrm{H}_{2}$ as a function of $R$. In region $\mathrm{I}$, where the bond is compressed, the system resembles a helium atom and all of the correlation is Type D. This is almost constant but decreases slightly as the bond lengthens and the electrons can avoid each other more easily. In region II, close to the equilibrium bond length $R_{e}$, the correlation energy increases but cannot be captured by UHF and is therefore Type B. In region III, which lies beyond the triplet instability, UHF is able to capture some of the correlation energy, which is therefore Type A. As the bond lengthens further, the fraction of Type A increases at the expense of Type B. At such stretched bond lengths, Type D correlation exists as dispersion and continues to decrease with increasing bond length. Finally, at dissociation, the HOMO and LUMO become degenerate and all of $E_{\text {corr }}$ can be captured by UHF. The presence of three types of electron correlation helps to explain the "unusual complexity"41 of the Pople correlation energy in Fig. 3.

\section{CONCLUSIONS}

In stretched $\mathrm{H}_{2}$, UHF can capture nearly all of $E_{\text {corr }}$. In the Be-like ions with $Z>4.5$, despite a linear growth in $E_{\text {stat }}$ with $Z$, UHF cannot capture any of $E_{\text {corr }}$. This puzzling difference can be rationalized by recognizing that there are two flavors of static correlation energy: Type A which results from an absolute near-degeneracy and which can be captured by UHF, and Type B which results from a relative near-degeneracy and which cannot be captured by UHF. At large $R$, the correlation energy in $\mathrm{H}_{2}$ is dominated by Type A correlation. At large $Z$, the correlation energy of the Be-like ions is dominated by Type B correlation. This unambiguous categorization of the static correlation energy will prove useful in the analysis of electron correlation and the development of new correlated approaches.

\section{ACKNOWLEDGMENTS}

J.W.H. thanks P.-F. Loos for valuable discussions and the National Science and Engineering Research Council of 
Canada for funding. P.M.W.G. thanks the NCI National Facility for supercomputer time and the Australian Research Council (Grants Nos. DP0771978 and DP0984806).

${ }^{1}$ A. Szabo and N. S. Ostlund, Modern Quantum Chemistry (Dover, New York, 1996)

${ }^{2}$ R. G. Parr and W. Yang, Density-Functional Theory of Atoms and Molecules (Clarendon Press, Oxford, 1989).

${ }^{3}$ A. M. K. Muller, Phys. Lett. A 105, 446 (1984).

${ }^{4}$ S. Goedecker and C. J. Umrigar, Phys. Rev. Lett. 81, 866 (1998).

${ }^{5}$ P. Leiva and M. Piris, J. Chem. Phys. 123, 214102 (2005).

${ }^{6}$ P. M. W. Gill, D. L. Crittenden, D. P. O'Neill, and N. A. Besley, Phys. Chem. Chem. Phys. 8, 15 (2006).

${ }^{7}$ E. E. Dumont, D. L. Crittenden, and P. M. W. Gill, Phys. Chem. Chem. Phys. 9, 5340 (2007).

${ }^{8}$ D. L. Crittenden, E. E. Dumont, and P. M. W. Gill, J. Chem. Phys. 127, 141103 (2007).

${ }^{9}$ Y. A. Bernard, D. L. Crittenden, and P. M. W. Gill, Phys. Chem. Chem. Phys. 10, 3447 (2008).

${ }^{10}$ J. K. Pearson, D. L. Crittenden, and P. M. W. Gill, J. Chem. Phys. 130, 164110 (2009).

${ }^{11}$ S. R. White, Phys. Rev. Lett. 69, 2863 (1992).

${ }^{12}$ C. K.-L. Chan, J. Chem. Phys. 120, 3172 (2004).

${ }^{13}$ U. Schollwöck, Rev. Mod. Phys. 77, 259 (2005).

${ }^{14}$ K. A. Hallberg, Adv. Phys. 55, 477 (2006).

${ }^{15}$ W. A. Lester, L. Mitas, and B. Hammond, Chem. Phys. Lett. 478, 1 (2009).

${ }^{16}$ A. Lüchow, R. Petz, and A. Schwarz, Z. Phys. Chem. 224, 343 (2010).

${ }^{17}$ P.-O. Löwdin, Adv. Chem. Phys. 2, 207 (1959).

${ }^{18}$ D. K. W. Mok, R. Neumann, and N. C. Handy, J. Phys. Chem. 100, 6225 (1996).

${ }^{19}$ T. Kato, Comm. Pure. Appl. Math 10, 151 (1957).

${ }^{20}$ F. London, Z. Phys. 63, 245 (1930).

${ }^{21}$ J. H. Lenthe, J. G. C. M. D.-V. D. Rijdt, and F. B. V. Duijneveldt, Adv. Chem. Phys. 69, 521 (1987).

${ }^{22}$ D. G. Truhlar, J. Comput. Chem. 28, 73 (2007).

${ }^{23}$ W. D. Laidig and H. F. Schaefer III, J. Chem. Phys. 74, 3411 (1981).

${ }^{24}$ P. Borowski, K. Andersson, P.-Å. Malmqvist, and B. O. Roos, J. Chem. Phys. 97, 5568 (1992).
${ }^{25}$ X. Li and J. Paldus, J. Chem. Phys. 110, 2844 (1999).

${ }^{26}$ K. J. Miller and K. Ruedenberg, J. Chem. Phys. 48, 3450 (1968).

${ }^{27}$ J. S. Sims and S. Hagstrom, Phys. Rev. A 4, 908 (1971).

${ }^{28}$ C. F. Bunge, Phys. Rev. A 14, 1965 (1976).

${ }^{29}$ J. Komasa, J. Rychlewski, and K. Jankowski, Phys. Rev. A 65, 042507 (2002).

${ }^{30}$ E. Valderrama, E. V. Ludena, and J. Hinze, J. Chem. Phys. 110, 2343 (1999).

${ }^{31}$ J. Linderberg and H. Shull, J. Mol. Spectrosc. 5, 1 (1960).

${ }^{32}$ E. Clementi and A. Veillard, J. Chem. Phys. 44, 3050 (1966).

${ }^{33}$ E. P. Ivanova and U. I. Safronova, J. Phys. B 8, 1591 (1975).

${ }^{34}$ P. F. Loos and P. M. W. Gill, J. Chem. Phys. 131, 241101 (2009).

${ }^{35}$ P. F. Loos and P. M. W. Gill, J. Chem. Phys. 132, 234111 (2010).

${ }^{36}$ P. F. Loos and P. M. W. Gill, Phys. Rev. Lett. 105, 113001 (2010).

${ }^{37}$ P. F. Loos and P. M. W. Gill, Chem. Phys. Lett. 500, 1 (2010).

${ }^{38}$ J. A. Pople and R. K. Nesbet, J. Chem. Phys. 22, 571 (1954).

${ }^{39}$ R. Seeger and J. A. Pople, J. Chem. Phys. 66, 3045 (1977).

${ }^{40}$ N. S. Ostlund, J. Chem. Phys. 57, 2994 (1972).

${ }^{41}$ V. A. Rassolov, M. A. Ratner, and J. A. Pople, J. Chem. Phys. 112, 4014 (2000).

${ }^{42}$ K. Raghavachari, G. W. Trucks, J. A. Pople, and E. Replogle, Chem. Phys. Lett. 158, 207 (1989).

${ }^{43}$ J. A. Pople and J. S. Binkley, Mol. Phys. 29, 599 (1975).

${ }^{44}$ D. P. O'Neill and P. M. W. Gill, Mol. Phys. 103, 763 (2005).

${ }^{45}$ M. V. Ivanov, Phys. Lett. A 239, 72 (1998).

${ }^{46}$ C. C. J. Roothaan, Rev. Mod. Phys. 23, 69 (1951).

${ }^{47}$ G. G. Hall, Proc. R. Soc. London A 205, 541 (1951).

${ }^{48}$ MATHEMATICA version 7.0, Wolfram Research, Inc. Champaign, IL, 2008.

${ }^{49}$ M. W. Schmidt and K. Ruedenberg, J. Chem. Phys. 71, 3951 (1979).

${ }^{50}$ S. Wilson and D. M. Silver, J. Chem. Phys. 72, 2159 (1980).

${ }^{51}$ E. S. Kryachko and S. Wilson, Int. J. Quantum Chem. 93, 112 (2003).

${ }^{52}$ D. L. Cooper and S. Wilson, J. Chem. Phys. 77, 4551 (1982).

${ }^{53}$ T. Koga, S. Watanabe, K. Kanayama, R. Yasuda, and A. J. Thakkar, J. Chem. Phys. 103, 3000 (1995).

${ }^{54}$ The discrepancy between Ivanov's $\Delta E_{\mathrm{UHF}}$ value $\left(0.334 \pm 0.013 \mathrm{~m} E_{\mathrm{h}}\right)$ and ours $\left(0.294 \mathrm{~m} E_{\mathrm{h}}\right)$ arises from the absence of higher $(d, f, \ldots)$ functions in our calculations.

${ }^{55}$ T. Helgaker, P. Jorgensen, and J. Olsen, Molecular Electronic Structure (Wiley, New York, 2000).

${ }^{56}$ A. Okniński, Chem. Phys. Lett. 27, 603 (1974). 